\title{
Latest investigations on fluctuations, ELM filaments and turbulent transport in the SOL of ASDEX Upgrade
}

\author{
H W Müller ${ }^{1}$, J Adamek ${ }^{2}$, R Cavazzana ${ }^{3}$, G D Conway ${ }^{1}$, C Fuchs ${ }^{1}$, J P Gunn ${ }^{4}$, A Herrmann ${ }^{1}$, \\ J Horaček $^{2}$, C Ionita ${ }^{5}$, A Kallenbach ${ }^{1}$, M Kočan ${ }^{1}$, M Maraschek ${ }^{1}$, C Maszl $^{5}$, F Mehlmann ${ }^{5}$, \\ B Nold ${ }^{6}$, M Peterka $^{7}$, V Rohde ${ }^{1}, J_{\text {Schweinzer }}{ }^{1}, \mathrm{R} \mathrm{Schrittwieser}^{5}$, N Vianello ${ }^{3}$, E Wolfrum ${ }^{1}$, \\ M Zuin ${ }^{3}$, and the ASDEX Upgrade Team ${ }^{1}$ \\ ${ }^{1}$ Max-Planck-Institut für Plasmaphysik, EURATOM Association, 85748 Garching, Germany \\ ${ }^{2}$ Institute of Plasma Physics, Association EURATOM/IPP.CR, Prague, Czech Republic \\ ${ }^{3}$ Consorzio RFX, Associazione EURATOM-ENEA sulla Fusione, Padova, Italy \\ ${ }^{4}$ CEA, IRFM, F-13108 Saint-Paul-lez-Durance, France \\ ${ }^{5}$ Institute for Ion Physics and Applied Physics, University of Innsbruck, Association \\ EURATOM/ÖAW, Austria \\ ${ }^{6}$ Institut für Plasmaforschung, Universität Stuttgart, Stuttgart, Germany \\ ${ }^{7}$ Faculty of Mathematics and Physics, Charles University of Prague, Czech Republic
}

E-mail contact of main author: hans.werner.mueller@ipp.mpg.de

\begin{abstract}
This paper presents turbulence investigations in the scrape off layer (SOL) of ASDEX Upgrade in Ohmic, L-mode and H-mode discharges using electrostatic and electromagnetic probes. Detailed studies are performed on small scale turbulence and on ELM filaments. Simultaneous measurements of floating and plasma potential fluctuations revealed significant differences between these quantities. Large errors can occur when the electric field is extracted from floating potential measurements, even in Ohmic discharges. Turbulence studies in Ohmic plasmas show the existence of density holes inside the separatrix and blobs outside. Close to the separatrix a reversal of the poloidal blob propagation velocity occurs. Investigations on the Reynolds stress in the scrape-off layer show its importance for the momentum transport in L-mode while its impact for momentum transport during ELMs in H-mode is rather small. In the far SOL the electron density and temperature were measured during type-I ELMy H-mode at ASDEX Upgrade resolving ELM filaments. Strong density peaks and temperatures of several $10 \mathrm{eV}$ were detected during the ELM events. Additional investigations on the ions in the filaments by a retarding field analyzer indicate ion temperatures of 50-80 eV. ELMs expel also current concentrated in filaments into the scrape off layer. Furthermore discharges with small ELMs were studied. In $\mathrm{N}_{2}$ seeded discharges the type-I ELM frequency rises and the ELM duration decreases. For discharges with small type-II ELMs the mean turbulent radial particle flux is increased over the mean particle flux in type-I ELM discharges at otherwise similar plasma parameters.
\end{abstract}

PACS: 52.25.Xz, 52.35.Ra, 52.55.Fa

\section{Introduction}

Turbulence is a key player in the plasma edge. Turbulence causes particle, energy and momentum transport in the edge and the scrape-off layer (SOL). Small scale turbulence is also expected to generate large scale flows like zonal flows via the Reynolds stress [1]. These zonal flows are thought to cause a velocity shearing of the turbulent eddies and therefore to increase the Reynolds stress transferring more energy from the small scale to the large scale turbulence ([2] and references therein). This altogether leads to a complex interplay of small and large scale turbulent structures which is also related to the formation of transport barriers [3] and strong radial electric fields $\mathrm{E}_{\mathrm{r}}$ [4]. A related topic is the rotation of plasmas, which also affects the stability e.g. [5][6]. Since the external momentum input in future fusion devices will be limited the role of the intrinsic rotation will become more important. Although the mechanisms creating the intrinsic momentum and the related momentum transport are not well identified and understood, the processes in the SOL might be important. There are strong parallel flows in the SOL, e.g. [7][8] which are expected to couple to the confined plasma 


\section{Turbulence and filaments in the SOL}

rotation [9]. SOL turbulence is also a candidate to transport momentum from the SOL to the confined plasma and vice versa. Beside the small scale turbulence and zonal flows intermittent transport is in the focus of interest. In L-mode turbulent structures called blobs or filaments can carry a significant fraction of the particle and energy transport, and in H-mode

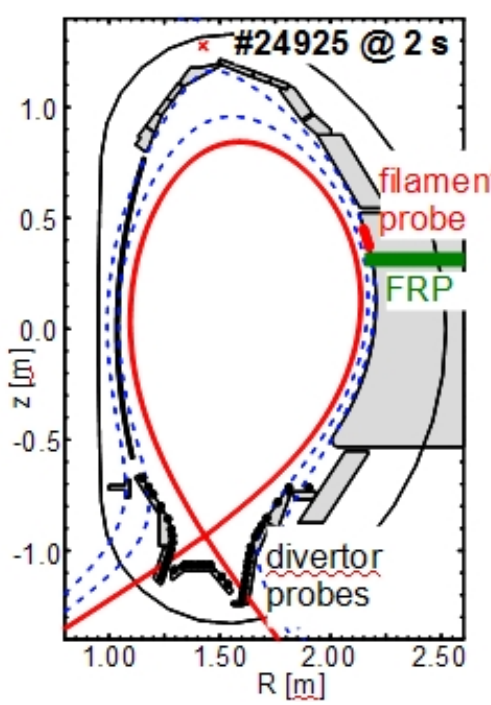

Figure 1: Poloidal cross section of ASDEX Upgrade showing a typical plasma shape and the poloidal positions of different Langmuir probe measurement locations. discharges large ELM filaments might even endanger the first wall of future devices due to high local heat loads [10]. Although there is large interest in turbulence occurring at the edge and in the SOL of tokamaks, many questions remained unanswered so far. This paper presents the latest progress in turbulence studies performed in the SOL of ASDEX Upgrade using electrostatic and electromagnetic reciprocating probes. The latest results of turbulence investigations with Doppler reflectometry on ASDEX Upgrade are presented in reference [3].

The paper is organized as follows: in section 2 the main features of the experimental setup are described. In the third section the experimental findings on small scale turbulence and the related electric fields in Ohmic and L-mode discharges are presented. Section 4 is devoted to new electron and ion temperature measurements in the SOL of ASDEX Upgrade. In section 5 we report about current filaments associated with ELMs. Turbulent transport properties in discharges with type-II ELMs are presented in section 6 . The influence of nitrogen $\left(\mathrm{N}_{2}\right)$ seeding onto type-I ELMs will be discussed in section 7 . The paper ends with a summary.

\section{Experimental setup}

ASDEX Upgrade [11] is a mid-size tokamak with a major radius of $R=1.65 \mathrm{~m}$ and a minor radius of $a=0.5 \mathrm{~m}$. If not stated otherwise the discharges presented in this paper were

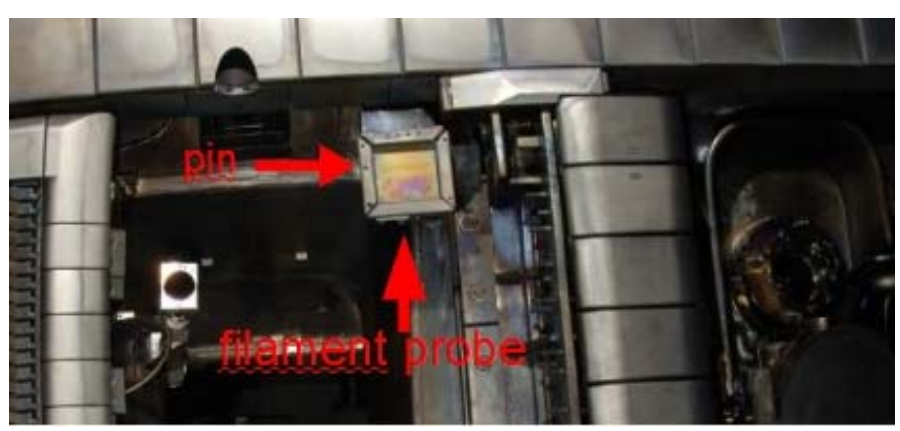

Figure 2: Filament probe in ASDEX Upgrade. The Langmuir probe pins are visible as black dots - one is highlighted by a red arrow. performed in single null configuration with the ion diamagnetic drift towards the active lower divertor (see also figure 1). For Langmuir probe measurements two reciprocating probes and fixed probes in the lower divertor were used. The poloidal probe locations are shown in figure 1 . The filament probe [12][13] rests in the limiter shadow about $40 \mathrm{~cm}$ above the equatorial plane (see figure 2). An electromagnetic drive pushes the probe carrying 9 Langmuir tips and a coil for measuring radial magnetic $B_{\mathrm{r}}$ fluctuations about $1 \mathrm{~cm}$ in front of the limiter for measurements. The pins are made of carbon and about $2 \mathrm{~mm}$ long and $2.4 \mathrm{~mm}$ thick. A fast reciprocating probe (FRP) [14] is located $31 \mathrm{~cm}$ above the equatorial plane. The FRP is equipped with a pneumatic drive allowing for a $100 \mathrm{~mm}$ stroke in $100 \mathrm{~ms}$. It can be equipped with different probe heads. For the investigations presented here several probe heads (see figure 3 ) were 


\section{Turbulence and filaments in the SOL}

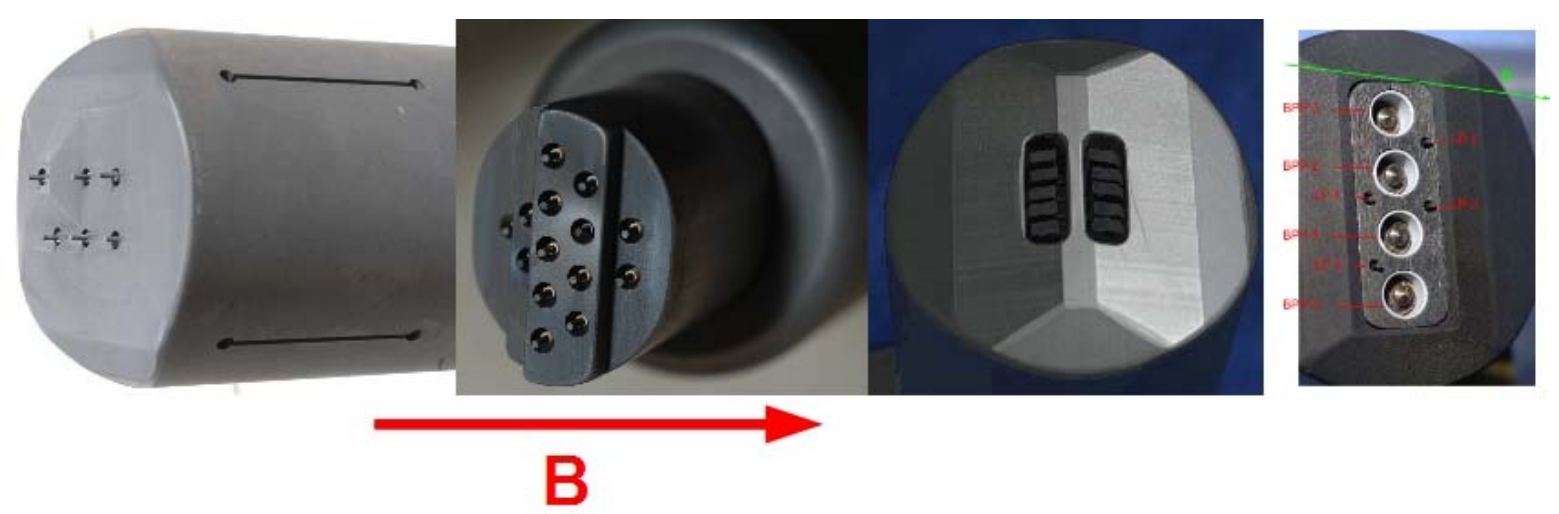

Figure 3: Probe heads for the FRP. From left to right, the probe for combined electrostatic and electromagnetic measurements (P6M1), the multi pin probe (MP), the high heat flux probe (HHF) with in-plane pins and the ball pen probe (BPP) with ball pen and Langmuir tips. The red arrow indicates roughly the direction of the magnetic field with respect to the probe pins.

exposed. For small scale turbulence measurements a multi pin probe head (MP) [15], a probe head which allows for combined electrostatic and electromagnetic measurements (P6M1) [16], and a ball pen probe (BPP) were used [17][18]. To measure ion energies, a retarding field analyzer (RFA) [19] was operated in ELMy H-mode. For investigations where high heat loads have to be expected a Mach type probe head with ten in-plane Langmuir probes (HHF) [20] similar to flush mounted probes was exposed to the plasma. Five pins were poloidally staggered at each side of the probe. The pin size is $4.2 \mathrm{~mm}$ in toroidal and $2 \mathrm{~mm}$ in poloidal direction. The pin surface is tilted by 14 degrees with respect to the toroidal direction. So, the active area perpendicular to the magnetic field is about $2 \mathrm{~mm}^{2}$. The multi-pin probe MP carries 14 protruding carbon pins distributed in three radially staggered planes, while the P6M1 has six protruding pins of which one is protruding by $3 \mathrm{~mm}$ compared to the others, and a triple coil (area about $50 \mathrm{~mm}^{2}$ ) measuring the time derivative of all three components of the magnetic field is located $20 \mathrm{~mm}$ behind the probe's front. All protruding pins have a length of $2 \mathrm{~mm}$ and a diameter of $0.9 \mathrm{~mm}$. The ball pen probe BPP was exposed to the SOL of

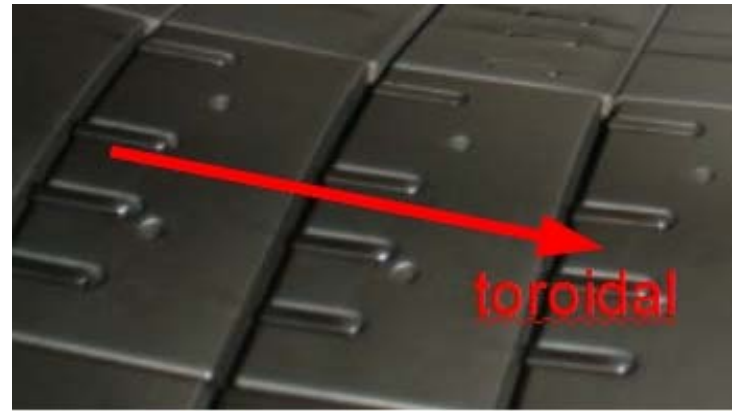

Figure 4: Langmuir probe tips in the outer lower divertor. They are flush mounted in the target tiles and made of tungsten. a mid size tokamak for the first time. It consists of four protruding pins and four retracted ball pen pins which allow for a direct measurement of the plasma potential $V_{\mathrm{pl}}$ [17]. The protruding pins are also $2 \mathrm{~mm}$ long with a diameter of 0.9 $\mathrm{mm}$, while the ball pen pins have a diameter of 4 $\mathrm{mm}$. It was the first time that a RFA was operated at ASDEX Upgrade. The RFA measures ion temperatures $T_{\mathrm{i}}$ and ion energy distributions. The probe can be operated as Mach probe to sample ions from both directions parallel to the magnetic field $B$ [19]. The data acquisition frequency of all electrostatic and electromagnetic measurements at the FRP and the filament probe is $2 \mathrm{MHz}$ with exception of the RFA collectors which data were sampled at $33 \mathrm{kHz}$. The $2 \mathrm{MHz}$ data acquisition system is equipped with a low pass filter of $700 \mathrm{kHz}$ band width. The sensors of the electromagnetic measurements presented in this paper have a bandwidth of $1 \mathrm{MHz}$. At high frequencies additional shielding by the carbon case of the probe head has to be expected. To diminish this effect there are slits in the carbon housing of the probe head (see figure 3 ). 


\section{Turbulence and filaments in the SOL}

For ELM investigations data from the Langmuir probes in the divertor were used. These probes are flush mounted in the divertor tiles and operated in triple probe configuration [21]. A typical probe size is about $20 \mathrm{~mm}$ in toroidal direction and $5 \mathrm{~mm}$ perpendicular in poloidal direction. A typical multi pin setup is shown in figure 4 . The data acquisition rate of this diagnostic is $33 \mathrm{kHz}$.

\section{Turbulence, potentials and electric field in Ohmic and L-mode discharges}

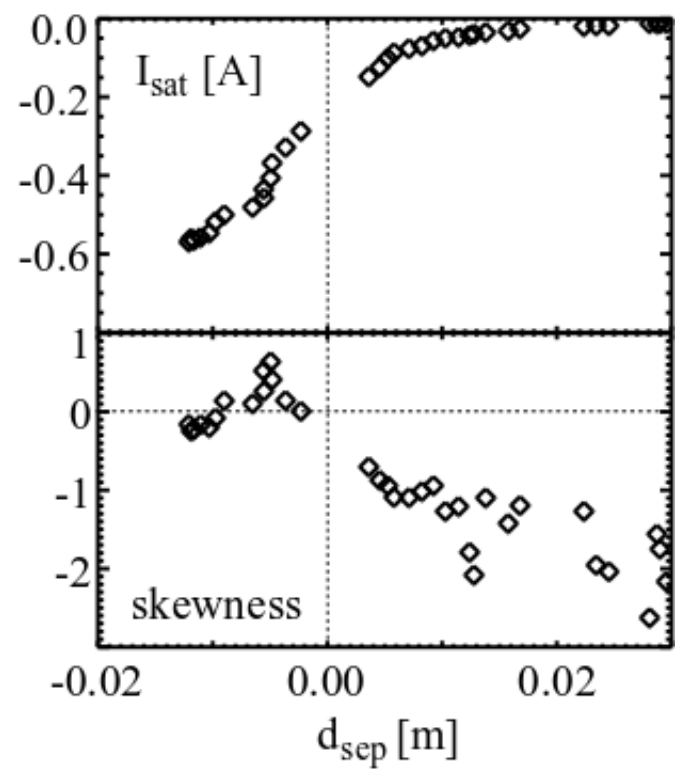

Figure 3: Profiles of the ion saturation current and the skewness of its fluctuations with respect to the distance to the separatrix $\left(d_{\text {sep }}\right)$ for \#24111 at 3.2s. The profiles are mapped onto the outer mid plane.

In a series of Ohmic discharges the basic properties of small scale turbulence were investigated in ASDEX Upgrade. The multi-pin probe was applied in Ohmic discharges [15]. The plasma current was $I_{\mathrm{p}}=0.8 \mathrm{MA}$, the toroidal magnetic field $B_{\mathrm{t}}=-2 \mathrm{~T}$, and the line averaged electron density $n_{\mathrm{e}, 1}=4-4.2 \times 10^{19} \mathrm{~m}^{-3}$. The ion saturation current fluctuations $I_{\text {sat,fl }}$ are expected to be dominated by electron density fluctuations $n_{\mathrm{e}, \mathrm{fl}}$ and therefore, $I_{\text {sat,fl }}$ is used as a measure of $n_{\mathrm{e}, \mathrm{fl}}$. The PDFs of $I_{\text {sat,fl }}$ clearly indicate that density blobs and holes are born close to the separatrix. While the blobs move outwards, the holes exist in a few $\mathrm{mm}$ wide area inside the separatrix. Figure 5 shows the radial distribution of hole and blob dominated areas. The data are taken while the reciprocating probe was moving inward. Ensembles of 2048 data points, which corresponds to a time interval of about $1 \mathrm{~ms}$ while the probe is moving about $1 \mathrm{~mm}$, were used to perform the statistical analysis. The boxcar window was shifted by 1024 data point in each step. In the range of $-1 \mathrm{~mm}$ to $+3 \mathrm{~mm}$ with respect to the separatrix were no data available since the applied voltage for the $\mathrm{I}_{\text {sat }}$ measurements was periodically switched to opposite sign. This is

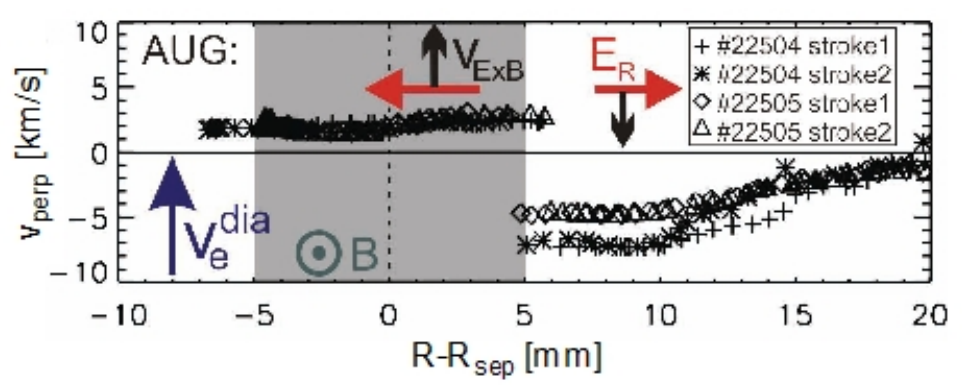

Figure 4: Radial profile of the perpendicular fluctuation propagation velocity mapped to the outer midplane. In the plot the direction of the magnetic field $B$, the radial electric field $E_{\mathrm{r}}$ (in red) and the diamagnetic electron drift direction $v_{\mathrm{e}}{ }_{\mathrm{e}}^{\mathrm{dia}}$ are indicated. The shaded area represents the uncertainty of the separatrix position. a standard procedure to terminate arcs which might occur on the probe tips. The steep $\mathrm{I}_{\text {sat }}$ gradient starting a few $\mathrm{mm}$ outside the separatrix reflects the density and temperature gradient in the plasma edge. The profiles have been shifted $5 \mathrm{~mm}$ inward with respect to the equilibrium reconstruction after comparing the $\mathrm{n}_{\mathrm{e}}$ and $\mathrm{T}_{\mathrm{e}}$ profiles of several diagnostics [15]. The skewness profile is clearly negative in the SOL (as the $\mathrm{I}_{\text {sat }}$ signal is) indicating the blobs are dominating. The blobs carry in 


\section{Turbulence and filaments in the SOL}

the SOL of L-mode discharges an outward radial particle flux. Just inside the separatrix there is a $10 \mathrm{~mm}$ wide region where the holes dominate indicated by the positive skewness. Since it is expected that blobs and holes are generated together in one process the place of birth has to be located close to the separatrix. At the separatrix or a few mm outside there is a strong shear layer as shown in figure 6 . The first $10 \mathrm{~mm}$ outside the separatrix are also the region of maximum normalized electron pressure indicating the area of maximum linear growth rate for instabilities which are driven by the pressure gradient [15]. The propagation velocity of the fluctuations in the poloidal plane perpendicular to $B$, shown in figure 6 for two Ohmic discharges at $n_{\mathrm{e}, 1}=2.6 \times 10^{19} \mathrm{~m}^{-3}$, is determined by cross correlation of two poloidally separated probe pins, both measuring $I_{\text {sat }}$ [22] [23]. A velocity shear is visible in the SOL accompanied by an abrupt flow reversal just outside the separatrix. The measurements do not indicate a smooth transition but a sudden jump between the two directions of motion which was well reproduced for four strokes. The profiles of the perpendicular velocity from probe cross correlation agree well with the velocity profile measured using the Doppler reflectometry technique [15], where the movement of turbulent density fluctuations is detected using the Doppler radar principle [3]. The frequency of the back scattered signal is Doppler shifted by $\omega=k_{\perp} u_{\perp}$, where $u_{\perp}=v_{\mathrm{E} \times \mathrm{B}}+v_{\mathrm{ph}}$ is the fluctuation propagation velocity composed out of the $E \times B$ drift and the fluctuation phase velocity $v_{\mathrm{ph}}$. The main difference is the smooth transition of the propagation direction seen by the Doppler reflectometry. This might be attributed to the different diagnostic techniques applied. The observation of the existence of blobs outside the separatrix and holes inside the separatrix and the shear layer corresponds well with the measurements at JET in Ohmic plasmas where the last closed flux surface touched the high field side limiter [24]. A difference occurs in the width of the shear layer. If the JET data are not mapped onto the mid plane, which is not stated in the reference, this can be (partly) attributed to a flux tube widening from the outer mid plane to the top of the machine. Also DIII-D reported that holes are dominating inside the separatrix and blobs outside with the transition close to the separatrix [25].

Doppler reflectometry is able to measure the radial electric field $E_{\mathrm{r}}=u_{\perp} B$ when the turbulence phase velocity $v_{\mathrm{ph}}$ is small compared to the $v_{\mathrm{ExB}}-$ which is generally the case in the edge. A common technique to derive $E_{\mathrm{r}}$ from Langmuir probe diagnostics are floating potential $\left(V_{\mathrm{flt}}\right)$ measurements at radially staggered Langmuir pins. The mean radial electric field $\left\langle E_{\mathrm{r}}\right\rangle$, where $\langle\ldots\rangle$ denotes a time average, is then calculated by $E_{\mathrm{r}}=\left(V_{\mathrm{flt}, 1}-V_{\mathrm{flt}, 2}\right) / d_{\mathrm{r}}$ with

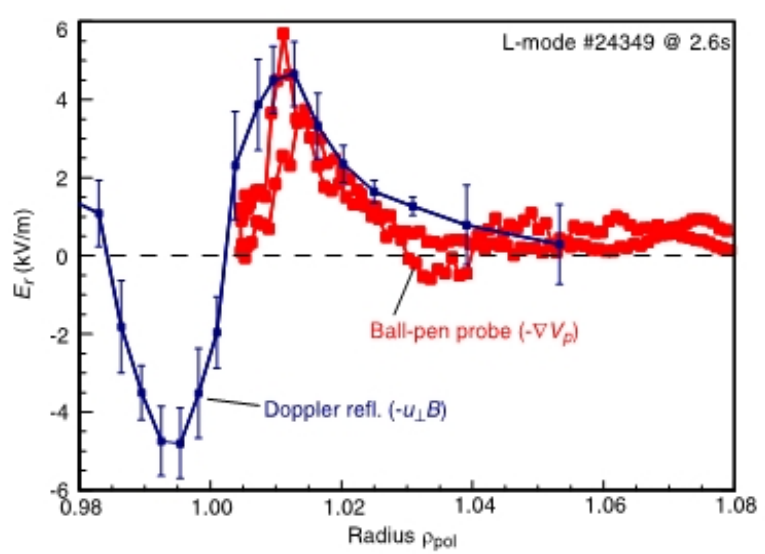

Figure 5: Comparison of $E_{\mathrm{r}}$ profile deduced from BPP and Doppler reflectometry. the radial tip distance $d_{\mathrm{r}}$ between the two pins. Plasma potential $V_{\mathrm{pl}}$ and floating potential $V_{\mathrm{flt}}$ are related via the electron temperature $T_{\mathrm{e}}$ : $V_{\mathrm{flt}}=V_{\mathrm{pl}}-\alpha / e T_{\mathrm{e}}$ with the elementary charge e and $\alpha \approx 2.8$ [14]. This offers a possibility to determine $\left\langle E_{\mathrm{r}}\right\rangle$ by measuring the $\left\langle V_{\mathrm{flt}}\right\rangle$ and electron temperature profile $\left\langle T_{\mathrm{e}}\right\rangle$ (e.g. taking the data from Thomson scattering or swept single probes) simultaneously and calculating $\left\langle E_{\mathrm{r}}\right\rangle=-\nabla_{\mathrm{r}}\left(\left\langle V_{\mathrm{flt}}\right\rangle+\alpha / e\left\langle T_{\mathrm{e}}\right\rangle\right)$. Both methods, with and without $T_{\mathrm{e}}$ term, were applied for the Ohmic discharge \#24111 (using Thomson scattering profiles for $T_{\mathrm{e}}$ ) and result in $\left\langle E_{\mathrm{r}}\right\rangle$ profiles which are not in agreement with the Doppler data in the steep gradient region 


\section{Turbulence and filaments in the SOL}

$\left(R-R_{\text {sep }}<1 \mathrm{~cm}\right)$. On the other hand, the propagation velocity of fluctuations deduced from cross-correlation analysis of the probe data agrees well with the velocity from the Doppler reflectometry. This points to the importance of electron temperature fluctuations $T_{\mathrm{e}, \mathrm{fl}}$ (on the spatial scale of the pin separation, several $\mathrm{mm}$ ) close to the separatrix. Using time averaged data from probes and Thomson scattering also fails to determine $V_{\mathrm{pl}}$ correctly. A strong influence of $T_{\mathrm{e}, \mathrm{fl}}$ on potential measurements was also reported from the ISTTOK tokamak [26].

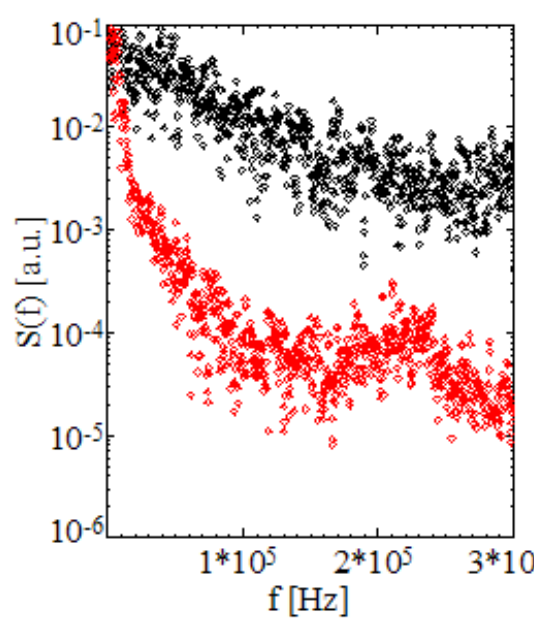

Figure 7: Comparison of the power spectrum of $V_{\text {flt }}$ (black) and $V_{\mathrm{pl}}$ (red).

The ball pen probe (BPP) is predicted to measure $V_{\mathrm{pl}}$ directly [17] which was successfully tested in small devices [27]. Recent experiments have shown that the BPP is also applicable to the SOL of mid-size tokamaks [18]. Figure 7 shows the comparison of an $E_{\mathrm{r}}$ profile directly calculated from $V_{\mathrm{pl}}$ measured by the BPP with a profile generated from the Doppler reflectometer data. The discharge (\#24349) was in L-mode at $I_{\mathrm{p}}=0.8 \mathrm{MA}, B_{\mathrm{t}}=-2.3 \mathrm{~T}, n_{\mathrm{e}, 1}=3.2 \times 10^{19} \mathrm{~m}^{-3}$. A heating power of $P_{\mathrm{NBI}}=1 \mathrm{MW}$ was applied by neutral beam injection. The two profiles agree which supports the assumption that in the SOL the poloidal motion of the density fluctuations is in fact governed by $v_{\mathrm{ExB}}$ while $v_{\mathrm{ph}}<<v_{\mathrm{E} \times \mathrm{B}}$. The comparison also gives confidence that BPPs can be used in the SOL of mid-size tokamaks to measure $V_{\mathrm{pl}}$ directly, at least in L-mode discharges. In a detailed analysis of ball pen probe characteristics it was found that there is a mild influence of $T_{\mathrm{e}}$ on the measured potential $V_{\mathrm{bpp}}=V_{\mathrm{pl}}-\alpha^{\prime} / e T_{\mathrm{e}}$ with $\alpha^{\prime}=0.6 \pm 0.3$ [18] which reduced the influence of $T_{\mathrm{e}}$ by roughly a factor of 5 compared to a standard Langmuir probe floating potential. In the

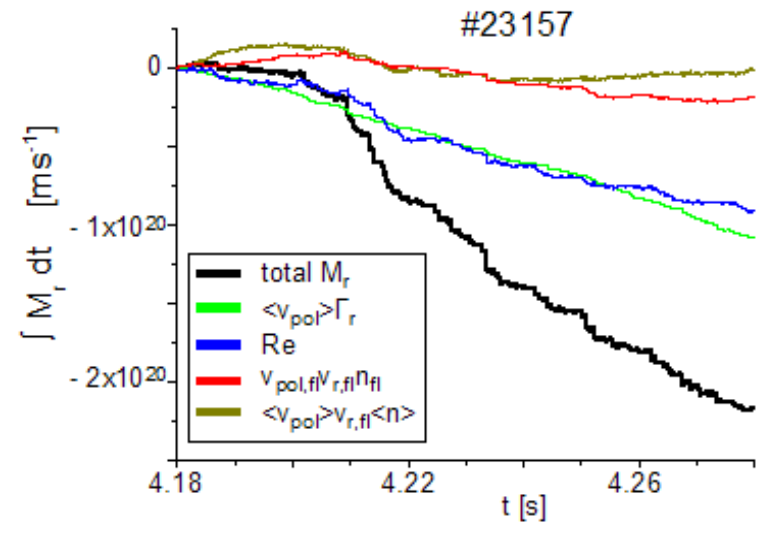

Figure 6: Integrated radial transport of poloidal momentum in a L-mode discharge. previous paragraph it was mentioned that due to electron temperature fluctuations $T_{\mathrm{e}, \mathrm{fl}}$ close to the separatrix $\left\langle E_{\mathrm{r}}\right\rangle$ cannot be correctly derived from $V_{\text {flt }}$ only. This is supported by a comparison of the $V_{\mathrm{pl}}$ (without $T_{\mathrm{e}}$ correction) and $V_{\text {flt }}$ power spectra measured simultaneously by a BPP in the SOL shown in figure 8. The data are taken in an L-mode discharge (\#24348) with the same plasma parameters as \#24349 and the probe was located $17 \mathrm{~mm}$ outside the separatrix at $z=0.31 \mathrm{~m}$. It is obvious that for frequencies above about $10 \mathrm{kHz}$ the turbulence level in $V_{\text {flt }}$ is significantly higher than in $V_{\mathrm{pl}}$. While the $V_{\mathrm{pl}}$ power spectrum shows a strong decrease with frequency the $V_{\text {flt }}$ spectrum stays rather flat. The same observation was made in H-mode in between ELMs [18].

ESEL code runs show that the length scale of $T_{\mathrm{e}, \mathrm{fl}}$ which is related to a density blob is much smaller than the spatial scale of the according $V_{\mathrm{pl}}$ turbulence [28]. The relative fluctuation 


\section{Turbulence and filaments in the SOL}

levels of $e V_{\mathrm{pl}, \mathrm{fl}}$ and $a T_{\mathrm{e}, \mathrm{fl}}$ are about the same. Both fluctuations are attributed to the same events, therefore moving with the same velocity. Consequently the smaller $T_{\mathrm{e}, \mathrm{fl}}$ structures allow for higher fluctuation frequencies in $V_{\mathrm{flt}, \mathrm{fl}}$ compared to $V_{\mathrm{pl}, \mathrm{fl}}$ while the amplitude of $a T_{\mathrm{e}, \mathrm{fl}}$ is of the same order as $e V_{\mathrm{pl}, \mathrm{fl}}$. In ESEL simulations for a TCV L-mode discharge a ratio of the

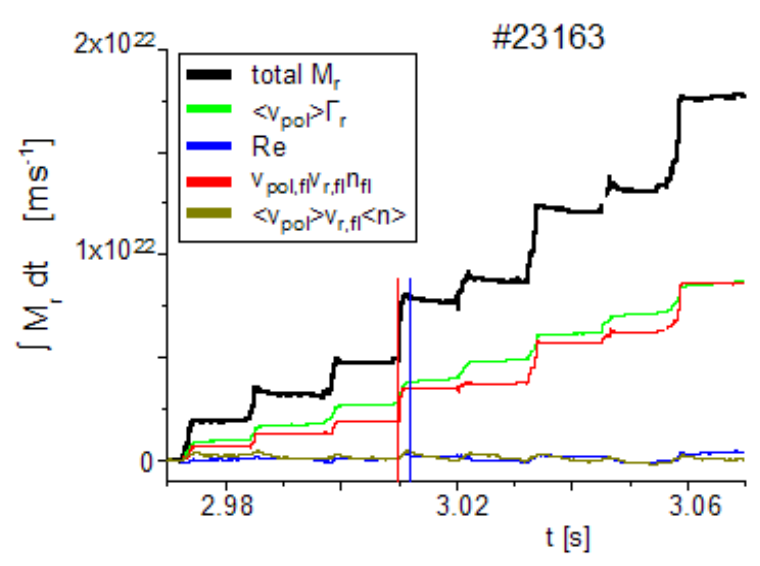

Figure 8: Time integrated radial transport of poloidal momentum during an $\mathrm{H}$-mode discharge. power spectral density of $V_{\mathrm{flt}, \mathrm{fl}}$ and $V_{\mathrm{pl}, \mathrm{fl}}$ was found which is similar to the ratio observed in ASDEX Upgrade [18]. Therefore, we conclude the $T_{\mathrm{e}, \mathrm{fl}}$ fluctuations contribute significantly to the difference in the power spectra of $V_{\text {flt,fl }}$ and $V_{\text {pl,fl. }}$. Albeit there might exist other additional contributions. An equivalent observation was made for ELM filaments in type-I H-mode discharges. The most reliable method to determine the radial propagation velocity of density fluctuations $v_{\mathrm{r}}$ is the cross correlation of radially staggered $I_{\text {sat }}$ measurements while calculating $v_{\mathrm{r}}$ from $E \times B$ using $V_{\mathrm{flt}}$ measurements to determine the poloidal electric field $E_{\mathrm{pol}}$ is strongly affected by the distance of the probe pins and the time interval for data averaging [29]. This indicates the observed turbulent structures and the spatial resolution of the diagnostic are of comparable size. On average $v_{\mathrm{r}}$ deduced from $E \times B$ using $V_{\text {flt }}$ data is lower than the value received from the cross correlation of Langmuir pins measuring $I_{\text {sat }}$.

A simultaneous measurement of $V_{\mathrm{pl}}$ and $V_{\mathrm{flt}}$ can be used to estimate $T_{\mathrm{e}}$ and to compare this with results from other diagnostics to test the approximation $V_{\mathrm{flt}}=V_{\mathrm{pl}}-\alpha / e T_{\mathrm{e}}$. In the Lmode discharge \#24349 a fair agreement between $T_{\mathrm{e}}$ derived from the simultaneously measured $V_{\mathrm{flt}}$ and $V_{\mathrm{pl}}$ (from the ball pen probe) potentials and $T_{\mathrm{e}}$ of a swept single probe was found in the far SOL for $R-R_{\text {sep }}>1 \mathrm{~cm}$ [18]. At the innermost position the $T_{\mathrm{e}}$ profile connects well to the Thomson scattering $T_{\mathrm{e}}$ profile. Summarizing the observations, it was found that close to the separatrix $\left\langle V_{\mathrm{pl}}\right\rangle$ cannot reliably be approximated by $\left\langle V_{\mathrm{flt}}\right\rangle+\alpha / e\left\langle T_{\mathrm{e}}\right\rangle$ using $\alpha=3$. $T_{\mathrm{e}}$ fluctuations cannot be neglected and seem to affect the measurement. Also in ELM filament studies the approximation is strongly limited since the substructure of the ELM filaments is of the same size as the measurement arrangement. For mean profiles in the SOL $R-R_{\mathrm{sep}}>1 \mathrm{~cm}$ at least in L-mode the relation $V_{\mathrm{flt}}=V_{\mathrm{pl}}-\alpha / e T_{\mathrm{e}}$ seems to hold ( $\mathrm{T}_{\mathrm{e}}$ from swept single probe). How strongly the floating potential fluctuations in the far SOL of L-mode discharges and inter-ELM periods of $\mathrm{H}$-mode discharges are influenced by $T_{\mathrm{e}, \mathrm{fl}}$ is not well 


\section{Turbulence and filaments in the SOL}

known. Further investigations will follow.

Investigations of turbulence induced radial transport of poloidal momentum have been performed with the combined electrostatic-electromagnetic probe P6M1 [30]. The probe was located in the far SOL during L-mode and H-mode discharges where the position was kept constant during the measurement. For these investigations it had to be assumed that the
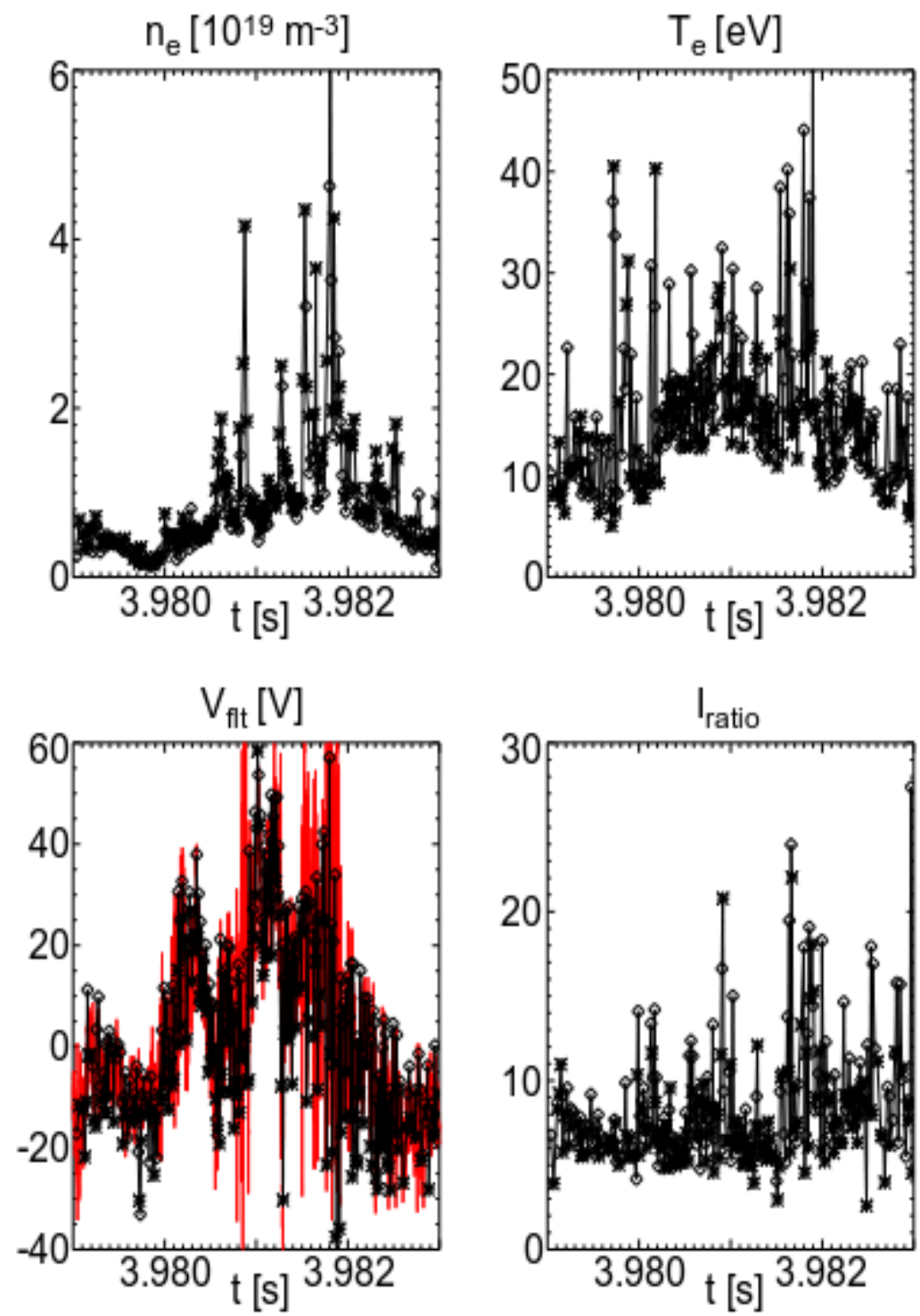

electric fields derived from $V_{\text {flt }}$ are only moderately affected by $T_{\mathrm{e}}$ fluctuations. In the far SOL of L-mode discharges this should be an acceptable approximation although for quantitative studies the influence of $T_{\mathrm{e}, \mathrm{fl}}$ has to be investigated. The measurement was performed in an Ohmic heated discharge, so there was no external momentum input. The plasma parameters were $I_{\mathrm{p}}=0.8 \mathrm{MA}$, $B_{\mathrm{t}}=-2.5 \mathrm{~T}, \quad$ and $n_{\mathrm{e}, 1}=4.9 \times 10^{19} \mathrm{~m}^{-3}$. The

probe location was $45 \mathrm{~mm}$ outside the separatrix (measured at $z=0.31 \mathrm{~m}$ ). The radial transport of poloidal momentum $M_{\mathrm{r}}=n_{\mathrm{e}} v_{\mathrm{r}} v_{\mathrm{pol}}$ can be split into the convective part due to the turbulent radial particle flux $\Gamma_{\mathrm{r}}\left\langle v_{\text {pol }}\right\rangle$, the Reynolds stress contribution $\Re=\left\langle n_{\mathrm{e}}\right\rangle v_{\mathrm{r}, \mathrm{fl}} v_{\text {pol,fl }}$, and a third order contribution $n_{\mathrm{e}, \mathrm{fl}} V_{\mathrm{r}, \mathrm{fl}} v_{\text {pol,fl }}$. Figure 9 shows the time integrated radial transport of poloidal momentum. It is obvious that the momentum transport is dominated by the convective part due to the turbulent radial particle transport $\Gamma_{\mathrm{r}}\left\langle v_{\mathrm{pol}}\right\rangle$ and

Figure 9: Electron density, temperature and floating potential fluctuations during an ELM. The ratio of ion and electron saturation current $\left(I_{\text {ratio }}\right)$ is also shown.

the Reynolds stress $\Re$. In a JET Ohmic limiter discharge the momentum flux was dominated by the Reynolds stress and the convective contribution rather small [24]. The transport is outward for a mean flow $\left\langle v_{\text {pol }}\right\rangle$ into the ion diamagnetic drift direction. The direction of the poloidal momentum agrees with the poloidal propagation velocity in the SOL shown in figure 6. The situation changes in $\mathrm{H}$-mode as shown in figure 10 . The parameters of the $\mathrm{H}$-mode 


\section{Turbulence and filaments in the SOL}

discharge \#23163 were $I_{\mathrm{p}}=0.8 \mathrm{MA}, B_{\mathrm{t}}=-2.5 \mathrm{~T}$, and $n_{\mathrm{e}, 1}=6.2 \times 10^{19} \mathrm{~m}^{-3}$, and additional heating power by neutral beam injection $P_{\mathrm{NBI}}=2.5 \mathrm{MW}$. The probe was placed $38 \mathrm{~mm}$ outside the separatrix. In H-mode the Reynolds stress term is small compared to the convective and the triple fluctuation term $n_{\mathrm{e}, \mathrm{fl}} v_{\mathrm{r}, \mathrm{fl}} v_{\mathrm{pol}, \mathrm{fl}}$. Also visible is that on average the transport of poloidal momentum shows the opposite sign than in the Ohmic case. The integrated value is dominated by the ELM bursts, while in between ELMs the radial transport of poloidal momentum is in the same direction as in the Ohmic discharge without external momentum input. Although the data in H-mode might be affected by rather large errors in the calculation of the electric fields, especially during ELMs, the general trend is expected to hold. Doppler measurements show in L-mode and H-mode about the same structure of $E_{\mathrm{r}}$, but the $E_{\mathrm{r}}$ shear close to the separatrix is strongly increased in H-mode [31]. The negative $E_{\mathrm{r}}$ dip is able to overcompensate the ion diamagnetic drift and allows for a positive poloidal plasma rotation as visible in figure 6 for an Ohmic discharge. Such a positive poloidal plasma rotation would be expected inside the separatrix. When an ELM filament hits the probe in the far SOL, the probe sees plasma originating from the pedestal region which includes the area of the $E_{\mathrm{r}}$ dip. One can speculate if it is possible that ELM filaments keep their momentum (direction) when crossing the shear layer. If this is possible this can explain the occurrence of the sign change in $M_{\mathrm{r}}$ with the ELM events. This is also in line with the observation that the 'local' Reynolds stress term is of less importance compared to the convective (particle transport with the ELM filament) and triple fluctuation term (ELM structure).

\section{ELM filament temperatures}

ELMs have a filamentary structure [32][33] and can transport significant energy to the first wall which is a potential hazard for next step fusion devices. Therefore, it is of great interest to gain knowledge on the electron $T_{\mathrm{e}}$ and ion $T_{\mathrm{i}}$ temperature development in filaments traveling across the SOL. For the first time in ASDEX Upgrade experiments were performed to measure $T_{\mathrm{e}}$ of individual ELM filaments with 5-10 is time resolution using single probes with fast swept bias voltage (HHF probe head). In L-mode discharges the comparison of slow $(\mathrm{kHz}$ range) and fast swept single probes indicated that at bias sweep frequencies of 50-100 $\mathrm{kHz}$ the probe characteristics still can be used to determine $n_{\mathrm{e}}$ and $T_{\mathrm{e}}$ without being affected by the polarization current caused by the potential difference between the intersected flux tube and the surrounding plasma [20]. In a type-I ELMy H-mode plasma (\#24925) with $I_{\mathrm{p}}=0.8 \mathrm{MA}, B_{\mathrm{t}}=-2.5 \mathrm{~T}, P_{\mathrm{NBI}}=3.6 \mathrm{MW}$, electron cyclotron heating power $P_{\mathrm{ECRH}}=1.5 \mathrm{MW}$, and $n_{\mathrm{e}, 1}=6.1 \times 10^{19} \mathrm{~m}^{-3}$, ELM filaments are detected with 10 ìs time resolution $45 \mathrm{~mm}$ outside the separatrix. Figure 11 shows $n_{\mathrm{e}}, T_{\mathrm{e}}, V_{\mathrm{flt}}$, and $I_{\text {ratio }}$ determined from the fast swept single probe (black data) during an ELM which starts at $t=3.980 \mathrm{~s}$. I Iratio $_{\text {is }}$ the ratio of electron to ion saturation current calculated in the fitting procedure of the $V-I$ characteristic. Data with an Iratio above 30 are removed since they are usually related to a bad fit of the characteristic. The asterisk (diamonds) indicate results while sweeping the bias voltage from positive (negative) to negative (positive) values. For comparison the $V_{\text {flt }}$ data of a nearby floating Langmuir pin is shown in red (data acquisition rate $2 \mathrm{MHz}$ ). A very good agreement is observed. The differences can mainly be attributed to the different time resolution. During the ELM $n_{\mathrm{e}}$ and $T_{\mathrm{e}}$ peaks of typically $n_{\mathrm{e}}=2-4 \times 10^{19} \mathrm{~m}^{-3}$ and $T_{\mathrm{e}}=30-40 \mathrm{eV}$, respectively, are detected at the probe. The filaments peak densities of $n_{\mathrm{e}}=2-4 \times 10^{19} \mathrm{~m}^{-3}$ are a substantial fraction of the pedestal top density of about $5 \times 10^{19} \mathrm{~m}^{-3}$. This indicates that the filaments originate from an area close to the pedestal top or further inside. The measured electron temperature is already 


\section{Turbulence and filaments in the SOL}

significantly lowered compared to the pedestal top temperature of about $600 \mathrm{eV}$. Starting at $3.9805 \mathrm{~s}$ there is a good correlation of the $n_{\mathrm{e}, \mathrm{fl}}$ and $T_{\mathrm{e}, \mathrm{fl}}$ peaks as shown in figure 11 (see also [20]). It is not yet clear, why there are no correlated fluctuations in the beginning of the ELM. In reference [20] a comparison is presented on a smoothed ELM $T_{\mathrm{e}}$ (25 is time resolution) derived from the BPP as well as from a fast swept single probe (100 kHz bias sweep). Both methods agreed well and delivered $T_{\mathrm{e}}=10-15 \mathrm{eV}$ during type-I ELM activity at the position of the outer limiter $55 \mathrm{~mm}$ outside the separatrix.

Investigations on $T_{\mathrm{i}}$ in ELM filaments indicated that $T_{\mathrm{i}}>T_{\mathrm{e}}$. In [34]. $T_{\mathrm{i}}$ was derived from the power load and $I_{\text {sat }}$ fall off lengths in the SOL during ELM activity delivering $T_{\mathrm{i}}=30-60 \mathrm{eV}$. This is supported by the first RFA data from ASDEX Upgrade showing $T_{\mathrm{i}}=50-80 \mathrm{eV}$ in the far SOL when ELM filaments arrive at the probe [19]. This ELM filament $T_{i}$ is consistent with current ELM transport models [35][36]. There is strong evidence that ion impact energies exceed $160 \mathrm{eV}$ in the far SOL during ELMs [19] which fits to the observed $T_{\mathrm{i}}$. The ion energy data are consistent with measurements from JET [36], raising concerns on the first wall life time in future fusion devices when unmitigated ELMs are present.

\section{Current Filaments}

Experiments indicated that ELMs do not only release particles and energy from the confined plasma but also current. In previous experiments at ASDEX Upgrade pick up coils were used to detect currents related to the ELM events. Details on this investigation can be found in [10]. The magnetic fluctuations detected during ELMs have been explained by mode structures in the confined plasma carrying a bidirectional current. In recent experiments the probe head for combined electrostatic and electromagnetic measurements P6M1 allowed for a simultaneous measurement of the fluctuations in all three components of $B$ with enhanced

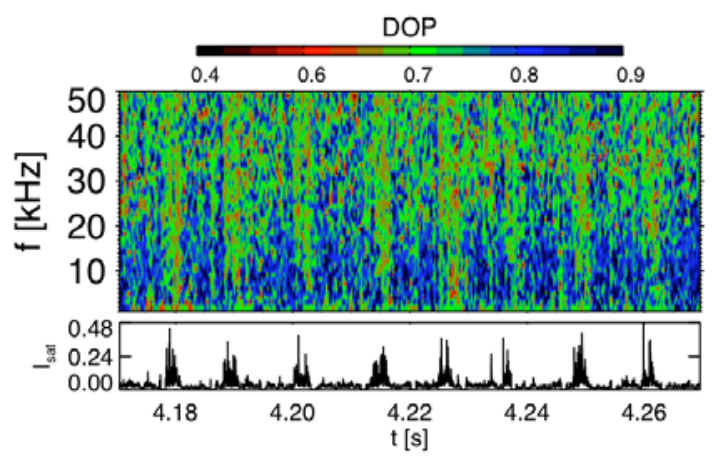

Figure 10: Top: DOP analysis of magnetic signature. Bottom: $I_{\text {sat }}$ signal indicating ELM activity. spatial resolution compared to the previous experiments [16] [37] [38]. Also the applied analysis methods were more advanced. Figure 12 shows the degree of polarization (DOP) during a type-I ELMy H-mode (\#23159). The plasma parameters are $I_{\mathrm{p}}=0.8 \mathrm{MA}$, $B_{\mathrm{t}}=-2.5 \mathrm{~T}, \quad P_{\mathrm{NBI}}=2.5 \mathrm{MW}, \quad$ and $n_{\mathrm{e}, 1}=6.1 \times 10^{19} \mathrm{~m}^{-3}$. The probe head was 12 $\mathrm{mm}$ in front of the limiter. The DOP is a test of the plane wave ansatz for the measured magnetic signature [38]. The analysis is done in the Fourier space. For each frequency it is calculated how well the magnetic signature can be related to a coherent fluctuations. This allows distinguishing between propagating modes and localized events. The reduction of the DOP with each ELM event (indicated by $\left.I_{\text {sat }}\right)$ shows that the magnetic signature of the magnetic fluctuations $B_{\mathrm{fl}}$ is no longer compatible to a plane wave and therefore most probably to be associated to a localized structure. The analysis of the magnetic signals reveals that the direction of the minimum $B_{\mathrm{fl}}$ is well aligned to the local magnetic field $B$. During ELM activity the $B_{\mathrm{fl}}$ components in the plane perpendicular to $B$ show a phase relation compatible to current filaments extended parallel to $B$ carrying a mono-polar current. These current filaments are located outside the separatrix at the magnetic low field side and show current densities up to $6 \mathrm{MAm}^{-2}$ [38]. Current densities of the same order have been measured in the pedestal region of H-mode plasmas at DIII-D 


\section{Turbulence and filaments in the SOL}

[39]. The measured filament current density is much higher than the ion saturation current $I_{\text {sat }}$ density measured at the probe which is of the order of $0.1 \mathrm{MAm}^{-2}$. But the $I_{\text {sat }}$ current is limited by the sheath in front of the probe head. This is not the case for the filament current measurement by magnetic pick up probes inhibiting this direct comparison.

In the following several simple possibilities are discussed which allow for the high current density of the mono-polar filament current. One possibility is that the filaments are still connected to the confined plasma. Since high current densities were measured also for filaments in the far SOL close to the limiter this is not very likely. Heat load measurements during ELM activity indicated that ELM filaments hitting the outboard limiters in ASDEX Upgrade are detached from the confined plasma [34]. The current path can be closed via a return current in the vessel structure. This would require a large widening of the current carrying flux tube towards the wall by an order of magnitude. The flux tube widening would not be required for hot spots, but they are an exception and therefore not considered here. Another rather simple mechanism to generate high current densities can occur just when the filament detaches from the confined plasma. At this time the ions and electrons in the flux tube stream freely towards the wall parallel $B$ until the sheath potential builds up. Assuming the filament first attaches to the outer divertor then there is a unidirectional high electron
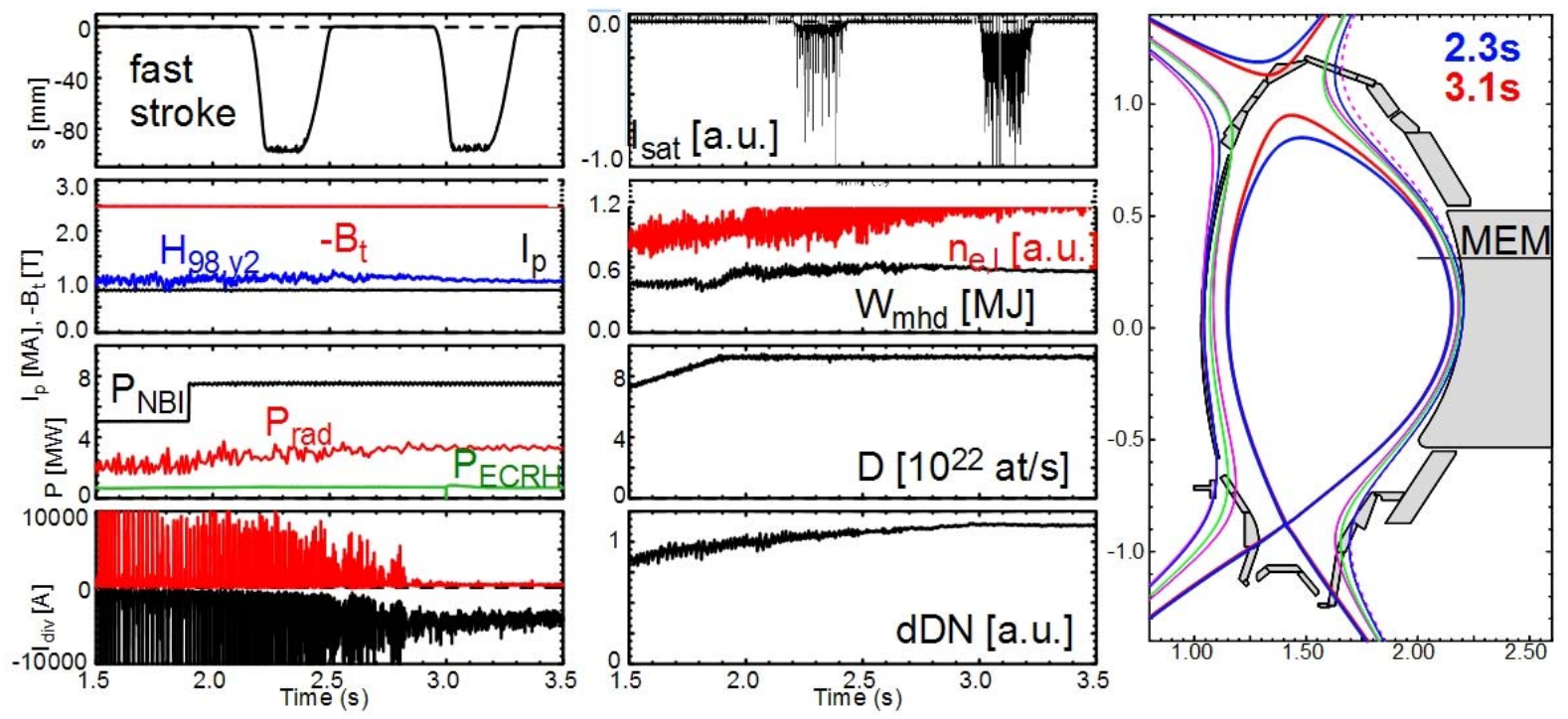

Figure 13: Main parameters of discharge \#25727 which shows a transition from type I to type II ELMy H-mode. From left top to right bottom: monitor signal of FRP strokes, $I_{\text {sat }}$ signal from Langmuir pin, $B_{\mathrm{t}}, I_{\mathrm{p}}$ and H98,y2, $n_{\mathrm{e}, 1}$ and stored energy $W_{\mathrm{mhd}}$, heating power, applied gas puff, thermocurrents in the inner (red) and outer (black) divertor acting as ELM monitor and closeness to double null. On the right hand side the plasma cross sections for the type-I ELM period (blue) and time-II ELM period (red).

current towards the outer divertor only. An upper limit for this current can be estimated by $j=e n_{\mathrm{e}} v_{\mathrm{e}, \mathrm{th}}$ where $e$ is the elementary charge, $n_{\mathrm{e}}$ the electron density in the filament and $v_{\mathrm{e}, \text { th }}$ the thermal electron velocity. With $n_{\mathrm{e}}=10^{19} \mathrm{~m}^{-3}$ and $T_{\mathrm{e}}=10 \mathrm{eV}$ the current density is already $j=1 \mathrm{MAm}^{-2}$. The data presented in the previous section showed that $n_{\mathrm{e}}$ and $T_{\mathrm{e}}$ can easily reach the assumed values. An upper bound for the time interval while this loss can occur can be estimated by $t_{\text {loss }} \sim L_{\mathrm{c}} / v_{\mathrm{e}, \text { th }}$ where $L_{\mathrm{c}}$ is the connection length from the mid plane to the divertor. Assuming a rather low $v_{\mathrm{e}, \text { th }}$ of $10^{6} \mathrm{~m} / \mathrm{s}$ which corresponds to a filament $T_{\mathrm{e}}$ of $10 \mathrm{eV}$ and a typical connection length of the order of $10 \mathrm{~m}$ the loss time interval is $t_{\text {loss }}=10^{-6} \mathrm{~s}$. This 


\section{Turbulence and filaments in the SOL}

is in contradiction to the fact that individual current filaments have been observed for $t \sim 10^{-4} \mathrm{~s}$. In fact the loss time interval has to be longer since the current carrying filament has to interact with Alfven waves and will bend field lines. Most probably this will be on cost of current density. Also the resistivity has not been taken into account. A complete analysis of the electromagnetic filament behaviour has to be carried out in full 3D geometry and is beyond the scope of this paper. A comprehensive qualitative description of the involved physics is presented in [40].

\section{Turbulent transport in type-II ELMy H-mode}

In a series of discharges a transition from type-I ELMy H-mode to type-II ELMy H-mode was induced by varying the closeness to double null (see figure 13). The global plasma parameters like $I_{\mathrm{p}}, B_{\mathrm{t}}$, heating power, radiated power $P_{\text {rad }}, \mathrm{H}$-factor, line averaged density $n_{\mathrm{e}, \mathrm{l}}$, stored energy $W_{\text {mhd }}$ and applied gas puff stayed constant. The presented data originate from discharge \#25727 with the following plasma parameters: $I_{\mathrm{p}}=0.8 \mathrm{MA}, B_{\mathrm{t}}=-2.5 \mathrm{~T}$, $P_{\mathrm{NBI}}=7.5 \mathrm{MW}, P_{\mathrm{ECRH}}=0.8 \mathrm{MW}$, and $n_{\mathrm{e}, 1}=5.5 \times 10^{19} \mathrm{~m}^{-3}$. In figure 13 it is already visible that
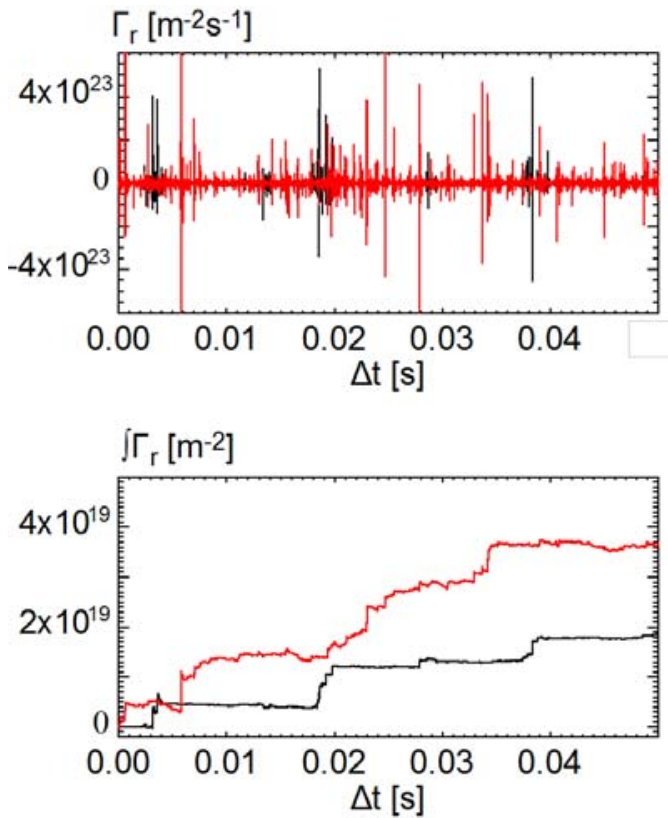

Figure 14: Top: time trace of the turbulence induced radial particle flux for a type-I ELMy (black) and type-II ELMy (red) H-mode). Bottom: time integral of the radial flux. during the second FRP stroke within the type-II ELMy $\mathrm{H}$-mode $I_{\text {sat }}$ exhibits stronger and more frequent excursions than during the first stroke. The turbulence induced radial flux in both phases of the discharge is shown in figure 14 while the probe was about $65 \mathrm{~mm}$ outside the separatrix. In the type-I period (shown in black) well separated bursts indicate the ELMs. In the type-II ELM period (red) the transport bursts are much more frequent in time. The time integrated radial particle flux clearly shows that the average particle flux is significantly higher during the type-II ELMy H-mode (red) than in the type-I ELMy H-mode although energy confinement and density are about the same. In both cases the particle flux is directed outward. In the type-II ELMy phase still rather large transport events can occur in the outer mid plane far SOL. This is visible by the steps in the integrated particle transport. Since the poloidal electric field $\mathrm{E}_{\mathrm{pol}}$ again had to be determined from $\mathrm{V}_{\text {flt }}$ there might be a rather large error in the amplitude but the general trends are expected to be robust.

\section{ELM characteristics in nitrogen seeded discharges}

During the recent years the plasma facing components in ASDEX Upgrade were fully covered by tungsten ([41][42] and references therein). With the first boronization of the machine with full tungsten walls the intrinsic radiation level significantly dropped and the power loads to the divertor could exceed the power limit for the tungsten coatings on the divertor tiles [43]. Therefore, feedback controlled nitrogen $\left(\mathrm{N}_{2}\right)$ seeding became a standard tool for radiative cooling in discharges with high heating power [44]. Nitrogen seeding not only resulted in an acceptable electron temperature in the divertor but also improved plasma performance [45]. Here, we focus on the influence on ELM properties and the transport onto the first wall. 


\section{Turbulence and filaments in the SOL}

The investigations were executed in deuterium H-mode discharges (\#23967-9, \#23979-84, \#24161-63, and \#24166) with $I_{\mathrm{p}}=1 \mathrm{MA}, B_{\mathrm{t}}=-2.5 \mathrm{~T}$ and line averaged densities of $n_{\mathrm{e}, 1}=8-9.5 \times 10^{19} \mathrm{~m}^{-3}$. In all discharges an additional heating of $P_{\mathrm{ECRH}}=1.5 \mathrm{MW}$ was applied and $P_{\mathrm{NBI}}=5-7.5 \mathrm{MW}$ was injected. The line averaged density required a rather high gas puff of $\Gamma_{\mathrm{D}}=5.8-9.1 \times 10^{21} \mathrm{~s}^{-1}$. The high gas puff at moderate heating powers lead to a rather low $\mathrm{H}$-factor of about $0.8-0.95$ (98Py2 scaling). In all $\mathrm{N}_{2}$ seeded discharges presented in this paper the set value for the divertor temperature was $3.0 \mathrm{eV}$. The $\mathrm{N}_{2}$ injection rate was in the range of $0.5-2 \times 10^{21} \mathrm{~s}^{-1}$ and the $\mathrm{N}$ concentration in the plasma was in the low $\%$ range. The feedback
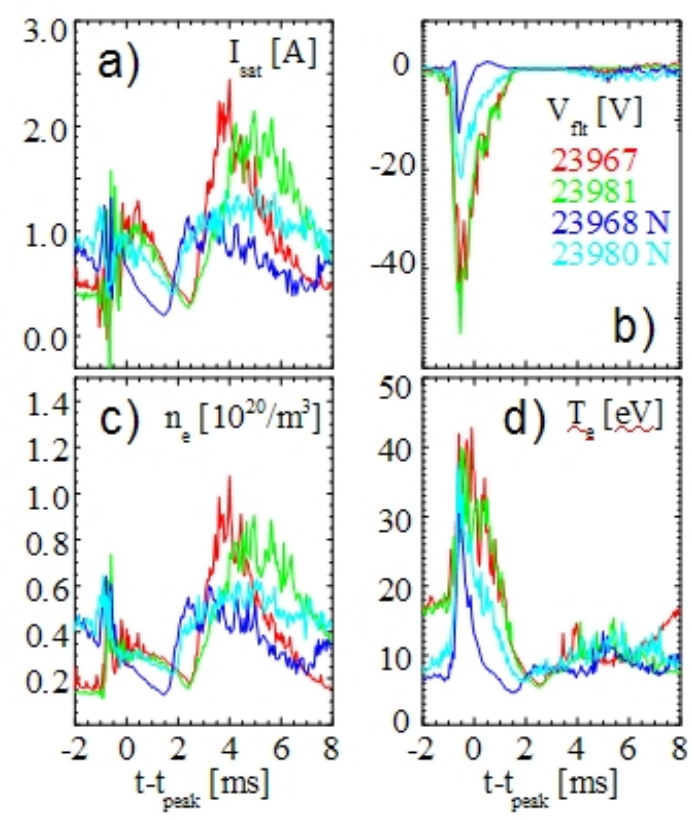

Figure 15: Main plasma parameters in the outer divertor $25 \mathrm{~mm}$ outside the separatrix during an ELM. The signals are conditional averaged. Shown are a) the ion saturation current $\left.I_{\mathrm{sat}}, b\right)$ the floating potential $V_{\mathrm{flt}}, c$ ) the electron density $n_{\mathrm{e}}$ and d) the electron temperature $T_{\mathrm{e}}$. The red and green lines indicate reference scenarios, the cyan curves show a discharge with $\mathrm{N}_{2}$ seeding in the main chamber and blue a discharge with $N_{2}$ seeding in the PFR.

decrease of the energy loss fraction per ELM $\Delta W_{\text {mhd }} / W_{\text {mhd }}$. This occurs either because of a clear reduction of $\Delta W_{\text {mhd }}$ at only slightly changed $W_{\text {mhd }}$ or by an increase in $W_{\text {mhd }}$ at roughly unchanged losses $\Delta W_{\text {mhd }}$ (which is in general a rather unusual case). These more general observations were supported by Langmuir probe measurements in the outer divertor about 25 $\mathrm{mm}$ outside the separatrix. This corresponds to $6 \mathrm{~mm}$ outside the separatrix in the outer mid plane In figure 15 the basic properties $I_{\mathrm{sat}}, V_{\mathrm{flt}}, T_{\mathrm{e}}$ and $n_{\mathrm{e}}$ are shown for two unseeded discharges in red and green, a discharge with $\mathrm{N}_{2}$ seeding in the PFR (blue) and one discharge with seeding in the main chamber (cyan). The measurement was performed with a triple probe and the conditional averaging technique was applied. The ELM induced $I_{\text {sat }}$ rise at the control acts on a time averaged divertor temperature which is determined by the inter ELM period. The total radiation $P_{\text {rad }}$ rose by $15 \%$ when $\mathrm{N}_{2}$ was injected in the main chamber and by $40 \%$ for injection into the private flux region (PFR). In discharges with higher additional heating power a clear increase in the stored energy $W_{\text {mhd }}$ was observed with $\mathrm{N}_{2}$ puffing, but there was a slight increase only in the discharges presented here due to the rather high $\mathrm{D}_{2}$ puff rate at moderate heating power. $W_{\text {mhd }}$ increased only by $5 \%$ when puffing $\mathrm{N}_{2}$ into the main chamber and by $10 \%$ for seeding in the PFR. In \#23979 the $\mathrm{N}_{2}$ injection did not change the total radiation of the plasma compared to the unseeded case and $W_{\text {mhd }}$ stayed about unchanged. So, there seems to be a direct relation between increase of $P_{\text {rad }}$ and $W_{m h d}$. This is confirmed by a study on the influence of the effective charge number on the confinement in $\mathrm{N}_{2}$ seeded discharges [46].

Independent of the seeding location the ELM frequency $f_{\mathrm{ELM}}$ rose by $20-50 \%$. Taking the ELM induced tungsten influx into the divertor as a measure for the ELM activity a reduction of the ELM duration from 3 to $2 \mathrm{~ms}$ occurs with $\mathrm{N}_{2}$ seeding into the PFR while main chamber seeding caused only a minor reduction of the ELM duration by $10 \%$. The nitrogen seeding independent of the seeding location causes a 


\section{Turbulence and filaments in the SOL}

beginning seems to be about the same in all cases although strongly affected by fluctuations. The duration of the $I_{\text {sat }}$ pulse is slightly reduced for the main chamber seeding and strongly reduced when injecting $\mathrm{N}_{2}$ into the PFR. The second $I_{\text {sat }}$ peak about 4-5 ms after the ELM event is rather typical for the all-tungsten ASDEX Upgrade and has already been discussed before [47][48]. This additional $I_{\text {sat }}$ peak in the inter-ELM period of the ELM cycle occurs in the high recycling regime of the divertor. It is related to the upstream plasma parameters. During the ELM cycle the density profile in the pedestal recovers within 4 ms while the edge temperature profile still develops [49]. These different recovery time scales most probably allow for a transient peak in the ratio of upstream density and temperature which, using a two point model, translates into a transient secondary $\mathrm{I}_{\text {sat }}$ peak at rather low $\mathrm{T}_{\mathrm{e}}$ [47]. The ELM

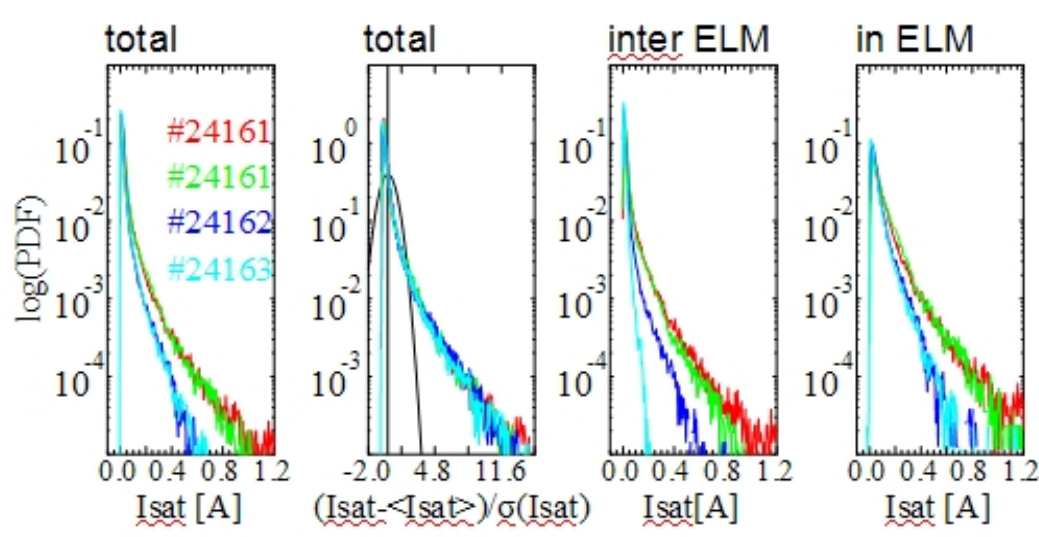

Figure 16: PDFs of the $I_{\text {sat }}$ signal at the filament probe. From left to right: PDF of total signal, $P D F$ of $I_{\text {sat }}$ fluctuation normalized to standard deviation, PDF in between ELMs and $P D F$ for $I_{\text {sat }}$ measurements in ELMs only. The red and green lines indicate the reference case without seeding. The cyan and the blue line are discharges with $\mathrm{N}_{2}$ seeding in the PFR. related density increase seems to follow the $I_{\text {sat }}$ signal. The floating potential peak is clearly decreased in amplitude when applying $\mathrm{N}_{2}$ where again seeding in the PFR is more effective. Also the $V_{\text {flt }}$ fall-off time seems to decrease with seeding into the PFR. This is related to the $T_{\mathrm{e}}$ behavior during the ELM. Injecting $\mathrm{N}_{2}$ also reduces the $T_{\mathrm{e}}$ pulse during the ELM. Especially the duration of the $T_{\mathrm{e}}$ pulse is reduced. The influence is more pronounced with $\mathrm{N}_{2}$ seeding into the PFR. In this case also a clear reduction of

the peak temperature is visible. These observations agree well with the shorter tungsten influx from the divertor tiles associated with the ELM. This influx is mainly caused by the $T_{e}$ rise due to an ELM. These findings fit well to the previous observations of reduced heat load and shunt currents during ELMs [50].

The filament probe was also used for turbulence studies in $\mathrm{N}_{2}$ seeded and unseeded H-mode discharges. Three probe pins were biased to measure $I_{\text {sat }}$ just in front of the outer limiter. The investigations were performed in discharges with an additional heating power of $P_{\mathrm{NBI}}=5 \mathrm{MW}$ and the seeding was performed in the PFR. With $\mathrm{N}_{2}$ seeding $I_{\text {sat }}$ decreased by $30-$ $50 \%$ in between ELMs as well as during ELMs. The reduction of the $I_{\text {sat }}$ level during ELMs seems not to be an effect of the ELM frequency $f_{\mathrm{ELM}}$ only. In the unseeded discharge \#24161 $f_{\text {ELM }}$ varies significantly but the $I_{\text {sat }}$ probability distribution function (PDF) stayed unchanged. Looking at the PDFs of the $I_{\text {sat }}$ fluctuations (figure 16) it is obvious that in the unseeded discharges (red and green) the probability for the occurrence of large events is higher than in $\mathrm{N}_{2}$ seeded discharges (blue and cyan). This observation holds during ELMs as well as in between ELMs. The PDFs verify the expectation that there are more large $I_{\text {sat }}$ events during the ELMs than in between. This is already reported from other experiments [51]. The reduction of the probability of large $I_{\text {sat }}$ events is of great interest since these events are related to large filaments carrying a rather high energy and might be able to harm the first wall in future fusion devices. The reduction of $t_{\mathrm{ELM}}$ with seeding is related to a reduction of the number of filaments per ELM. Normalizing $I_{\text {sat }}$ by using $\left(I_{\text {sat }}-\left\langle I_{\text {sat }}\right\rangle\right) / \sigma\left(I_{\text {sat }}\right)$, where $\sigma$ denotes 


\section{Turbulence and filaments in the SOL}

the standard deviation, yields that all normalized PDFs with or without $\mathrm{N}_{2}$ seeding are identical, showing a strong deviation from a Gaussian distribution and indicating strong intermittency caused by blobs/filaments. The agreement in the normalized PDFs indicates that the transport mechanism remains unchanged, but the level is reduced with $\mathrm{N}_{2}$ seeding.

\section{Summary}

The origin of blobs and holes in Ohmic discharges was identified to be close to the separatrix. $T_{\mathrm{e}}$ fluctuations cannot in general be neglected in Ohmic and L-mode discharges at ASDEX Upgrade when investigating turbulence, especially close to the separatrix. Close to the separatrix neglecting $T_{\mathrm{e}, \mathrm{fl}}$ might cause errors when $V_{\mathrm{pl}}$ is calculated from $V_{\text {flt }}$ even when using time averaged values. Electric fields during ELM activity in H-mode derived from $V_{\text {flt }}$ can also be affected by $T_{\mathrm{e}, \mathrm{fl}}$ causing rather large uncertainties. The poloidal momentum transport in the SOL was measured for the first time in ASDEX Upgrade showing the importance of the Reynolds stress in L-mode which is of comparable size as the convective momentum transport. In the H-mode time average a triple fluctuation term becomes comparable to the convective term and both are more important than the Reynolds stress. ELMs change the direction of momentum transport or the direction of the momentum which is transported radially. ELM filament resolved $T_{\mathrm{e}}$ and $n_{\mathrm{e}}$ measurements showed rather hot and dense ELM filaments (several $10 \mathrm{eV}$ and several $10^{19} \mathrm{~m}^{-3}$ ) in the far SOL while $T_{\mathrm{i}}$ is in the range 50-80 $\mathrm{eV}$. These values indicate that the ELM filaments originate from deep inside the pedestal. ELMs are associated with field aligned mono-polar current filaments carrying currents up to $\mathrm{kA}$. In type-II ELMy H-modes the outward radial particle flux is enhanced compared to type-I ELMy $\mathrm{H}$-mode at about the same global plasma parameters. $\mathrm{N}_{2}$ seeding shortens the ELM duration and the probability of the occurrence of large filaments is reduced.

\section{Acknowledgment}

The authors like to thank CEA for building a RFA in kind for operation at ASDEX Upgrade. This work was supported by the EFDA Task Agreement WP09-TGS-02c/01, the projects GA AV KJB100430901 of the Grant Agency of AS CR and the grant P19901 of the Austrian Science Funds. The content of the publication is the sole responsibility of its authors and does not necessarily represent the views of the European Commission or its services.

\section{References}

[1] Scott B., Phys. Lett. A 320 (2003) 53

[2] Diamond P.H., Itoh S.-I., Itoh K., Hahm T.S., Plasma Phys. Control. Fusion, 47 (2005) R35

[3] Conway G.D. et al. in Fusion Energy 2010 (Proc. 23 ${ }^{\text {rd }}$ Int. Conf. Geneva, 2010) (Vienna: IAEA) CD-ROM file EXC/7-1 and http://www-pub.iaea.org/mtcd/meetings/PDFplus/2010/cn180/cn180_papers/exc_7-1.pdf

[4] Terry P.W., Rev. Mod. Phys., 72 (2000) 109

[5] Strait E.J., Taylor T.S., Turnbull A.D., Ferron J.R., Lao L.L., Rice B., Sauter O., Thomson S.J., Wroblewski D., Phys. Rev. Lett., 74 (1995) 2483

[6] Politzer P.A., Nucl. Fusion, 48 (2008) 075001

[7] LaBombard B. et al., Nucl. Fusion 44 (2004) 1047

[8] Müller H.W., Bobkov V., Herrmann A., Maraschek M., Nauhauser J., Rohde V., Schmid A., Tsalas M., ASDEX Upgrade Team, J. Nucl. Mater. 363-365 (2007) 605

[9] Isoardi L., Ciraolo G., Chiavassa G., Haldenwang P., Serre E., Ghendrih Ph., Sarazin Y., Schwander F., Garbet X., Tamain P., J. Nucl. Mater. 390-391 (2009) 388 


\section{Turbulence and filaments in the SOL}

[10] Herrmann A. et al. in Fusion Energy 2008 (Proc. $22^{\text {nd }}$ Int. Conf. Geneva, 2008) (Vienna: IAEA) CD-ROM file EX/P6-1 and

http://www-naweb.iaea.org/napc/physics/FEC/FEC2008/html/index.htm

[11] Herrmann A., Gruber O., Fusion Science and Technology 44 (2003) 569

[12] Schmid A., Herrmann A., Rohde V., Maraschek M., Müller H.W., Rev. Sci. Instr., 78 (2007) 053502

[13] Schmid A., Herrmann A., Müller H.W., Plasma Phys. Control. Fusion, 50 (2008) 045007

[14] Rohde V., ASDEX Upgrade Team in Europhysics Conference Abstracts (Proc. of the 23rd EPS Conference on Plasma Physics, Kiev, 1996), Eds. Gresillon D., Sitenko A., Zagorodony A. (EPS, Geneva) Vol. 20C, part II (1996), 811

[15] Nold B., Conway G.D., Happel T., Müller H.W., Ramisch M., Rohde V., Stroth U., ASDEX Upgrade Team, Plasma Phys. Control. Fusion 52 (2010) 065005

[16] Ionita C. et al., J. Plasma Fusion Res. 8 (2009) 413

[17] Adamek J., Rohde V., Müller H.W., Herrmann A., Ionita C., Schrittwieser R, Mehlmann

F., Stöckel J., Horacek J., Brotankova J., J. Nucl. Mat. 390-391 (2009) 1114

[18] Adamek J. et al., Contr. Plasma Phys. 50 (2010) 853

[19] Kocan M. et al., Plasma Phys. Control. Fusion 53 (2011) 065002

[20] Müller H.W., Adamek J, Horacek J., Ionita C., Mehlmann F., Rohde V., Schrittwieser R., ASDEX Upgrade Team, Contr. Plasma Phys. 50 (2010) 847

[21] Weinlich M. and Carlson A., Contrib. Plasma Phys., 35 (1996) 53

[22] Nold B., diploma thesis, 'Comparison of Dimensionally Similar Turbulence in TJ-K and ASDEX Upgrade', IPP report 10/34, 2008, Max-Plack-Institut für Plasmaphysik, Garching

[23] Stroth U. et al. in Fusion Energy 2008 (Proc. 22 ${ }^{\text {nd }}$ Int. Conf. Geneva, 2008) (Vienna: IAEA) CD-ROM file EX/P4-23 and

http://www-naweb.iaea.org/napc/physics/FEC/FEC2008/html/index.htm

[24] Xu G.S. et al., Nucl. Fusion 49 (2009) 092002

[25] Boedo J.A. et al., Phys. Plasmas 10 (2003) 1670

[26] Schrittwieser R., Ionita C., Balan P., Silva C., Figueiredo H., Varandas C.A.F., Rasmussen J.J., Naulin V., Plasma Phys. Control. Fusion 50 (2008) 055004

[27] Adamek J. et al., Czech. J. Phys. 55 (2005) 235

[28] Horacek J., Adamek J., Müller H.W., Seidl J., Nielsen H., Rohde V., Mehlmann F., Ionita C., Havlickova E., ASDEX Upgrade Team, Nucl. Fusion, 50 (2010) 105001

[29] Kirk A., Müller H.W., Herrmann A., Kocan M., Rohde V., Tamain P., ASDEX Upgrade Team, Plasma Phys. Control. Fusion 53 (2011) 035003

[30] Mehlmann F. et al. in Europhysics Conference Abstracts (Proc. of the $37^{\text {th }}$ EPS Conference on Plasma Physics, Dublin, 2010) CD-ROM file P1.1064 and http://ocs.ciemat.es/EPS2010PAP/pdf/P1.1064.pdf

[31] Schirmer J., Conway G.D., Zohm H., and the ASDEX Upgrade Team, Nucl. Fusion 46 (2006) S780

[32] Eich T., Herrmann A., Neuhauser J., Phys. Rev. Lett. 91 (2003) 195003

[33] Kirk A., Wilson H.R., Counsell G.F., Akers R., Arenfs E., Cowley S.C., Dowling J., Lloyd B., Proce M., Walsh M. (MAST Team), Phys. Rev. Lett. 92 (2004) 245002

[34] Herrmann A. et al., J. Nucl. Mater 363-365 (2007) 528

[35] Fundamenski W., Plasma Phys. Control. Fusion 48 (2006) 106

[36] Pitts R.A., Fundamenski W., Erents S.K., Andrew Y., Loarte A., JET-EFDA Contributors, Nucl. Fusion 46 (2006) 82

[37] Martines E. et al., Plasma Phys. Control. Fusion 51 (2009) 124035

[38] Vianello N. et al., Phys. Rev. Lett. 106 (2011) 125002

[39] Thomas D.M., Leonard A.W., Lao L.L., Osborne T.H., Mueller H.W., Finkenthal D.F., 


\section{Turbulence and filaments in the SOL}

Phys. Rev. Lett. 93 (2004) 065003

[40] Xu G.S., Naulin V., Fundamenski W., Rasmussen J.J., Nielsen A.H., Wan B.N., Phys. Plasmas 17 (2010) 022501

[41] Neu R., Dux R., Geier A., Greuner H., Krieger K., Maier H., Pugno R., Rohde V., Yoon S.W., ASDEX upgrade Team, J. Nucl. Mater. 313-316 (2003) 116

[42] Neu R. et al., Plasma Phys. Control. Fusion 49 (2007) B59

[43] Herrmann A., Greuner H., Fuchs J.C., de Marne P., Neu R., ASDEX Upgrade Team, Phys. Scr. 2009 (2009) 014059

[44] Kallenbach A. et al., Plasma Phys. Control. Fusion 52 (2010) 055002

[45] Gruber O. et al., Nucl. Fusion 49 (2009) 115014

[46] Rathgeber S. et al., Plasma Phys. Control. Fusion, 52 (2010) 095008

[47] Wischmeier M., Kallenbach A., Chankin A.V., Coster D.P., Eich T., Herrmann A., Müller H.W., ASDEX Upgrade Team, J. Nucl. Mater. 363-365 (2007) 448

[48] Kallenbach A., Dux R., Eich T., Fischer R., Giannone L., Harhausen J., Herrmann A., Müller H.W., Pautasso G., Wischmeier M., ASDEX Upgrade Team, Nucl. Fusion 48 (2008) 085008

[49] Wolfrum E., Burckhart A., Fischer R., Hicks N., Konz C., Kurzan B., Langer B., Pütterich T., Zohm H., ASDEX Upgrade Team, Plasma Phys. Control. Fusion 51 (2009) 124057

[50] Kallenbach A. et al., Nucl. Fusion 49 (2009) 045007

[51] Ben Ayed N., Kirk A., Dudson B., Talents S., Vann R.G.L., Wilson H.R., MAST Team, Plasma Phys. Control. Fusion 51 (2009) 035016 